\title{
Sense of coherence modifies the effect of overtime work on men- tal health
}

\author{
Masanori OHta $^{1}$, Yoshiyuki Higuchi ${ }^{2}$, Hiroshi Yamato $^{1}$, Masaharu Kumashiro ${ }^{3}$ and \\ Hisamichi SUGIMURA ${ }^{4}$
}

${ }^{1}$ Department of Health Development, Institute of Industrial Ecological Sciences, University of Occupational and Environmental Health, Japan, ${ }^{2}$ Department of Education, Fukuoka University of Education, Japan, ${ }^{3}$ Emeritus Professor, University of Occupational and Environmental Health, Japan and ${ }^{4}$ Department of Ergonomics, Institute of Industrial Ecological Sciences, University of Occupational and Environmental Health, Japan

\begin{abstract}
Sense of coherence modifies the effect of overtime work on mental health: Masanori ОHтA, et al. Department of Health Development, Institute of Industrial Ecological Sciences, University of Occupational and Environmental Health, JapanObjectives: In the occupational health field, it is important to know how workload influences mental health. Overtime work and job strain appear to affect the mental health status of workers. Sense of coherence (SOC) may mediate the relationship between work stress and mental health. Since SOC represents a personal ability to manage psychological stressors, we hypothesized that a strong SOC would modify the adverse effect of an objective measure of overtime work on mental health. Methods: A total of 1,558 Japanese workers employed in an information technology company were asked to complete a 3-item SOC Questionnaire and 28-item General Health Questionnaire (GHQ) to assess mental health status. Workload was assessed by the actual amount of overtime work hours recorded by the company. Results: Multiple regression analysis revealed a main effect of overtime work $(\beta=0.08$, $p=0.0003)$ and SOC scores $(\beta=0.41, p<0.0001)$ on $\mathrm{GHQ}$ scores. There was a tendency toward interaction between overtime work and SOC scores $(\beta=0.05$, $p=0.051)$. Simple slope analysis supported this association $(-1 S D$ below the mean, simple slope $=0.04$, $S E=0.01, p<0.0001 ;+1 S D$ above the mean, simple slope $=0.01, S E=0.01, p=0.188)$. Conclusions: These results suggest that SOC buffers the mental health impacts of workload as measured by an objective index of overtime work, and should be considered when
\end{abstract}

Received Jul 11, 2014; Accepted Dec 31, 2014

Published online in J-STAGE Feb 7, 2015

Correspondence to: M. Ohta, Department of Health Development, Institute of Industrial Ecological Sciences, University of Occupational and Environmental Health, Japan, 1-1 Iseigaoka, Yahatanishi-ku, Kitakyushu, Fukuoka 807-8555, Japan (e-mail: ohta-msn@med. uoeh-u.ac.jp) assessing the effects of workload on mental health. (J Occup Health 2015; 57: 297-301)

Key words: Mental health status, Overtime work, Sense of coherence

Overtime work is a vitally important occupational health issue in developed countries, especially Japan, because the number of work-related mental disorder cases due to overtime work increases yearly ${ }^{1)}$. Previous research suggests that overtime work is associated with considerable mental and physical stress $^{2)}$. Conversely, Hobson and Beach reported the importance of determining psychological health by perceived workload compared with actual workload ${ }^{3)}$. As individuals may have widely differing perceptions of the same work, Hobson and Beach emphasized risk assessment, with perception being the key to translating hazards (actual stressors) into risk (perceived stressors). Thus, how one perceives stressors-that is, recognizing one's style of handling occupational stress-is important.

Sense of coherence (SOC) may mediate the relationship between work stress and mental health. Antonovsky defined SOC as "a global orientation that expresses the extent to which one has a pervasive, enduring though dynamic feeling of confidence"4). SOC refers to a person's sense of optimism and control over his/her environment, and it represents a personal ability to manage psychological stressors. A strong SOC is associated with positive perceptions of health. Theoretically, SOC consists of comprehensibility, manageability, and meaningfulness. "Comprehensibility" involves recognizing stress as understandable, "manageability" involves believing one has sufficient stress management resources, and "meaningfulness" involves believing life has mean- 
ing. Antonovsky described these components as "inextricably intertwined", suggesting that "successful coping depends on the SOC as a whole"4). Thus, we analyzed SOC as a whole.

SOC may mediate the translation of actual stressors, such as overtime work, into impaired psychological health. Research on models such as job-demand control and psychological workload due to time pressure showed that a higher SOC has a protective effect on the mental health status of workers ${ }^{5,6)}$. SOC might affect self-reported working time. However, no study has focused on the association of SOC with an objective measure of overtime work. We hypothesized that a strong SOC would modify the adverse effects of overtime work on mental health. This study estimated the buffering effect of SOC on the association between objective overtime work (calculated with company payroll records) and mental health in a Japanese information technology (IT) company.

\section{Subjects and Methods}

The subjects were 1,667 workers of an IT company located in Tokyo, Japan. The survey was advertised and administered through the company's internal website. We protected the privacy of the participants by limiting personal data access to an occupational doctor only. Furthermore, we emphasized that participation was voluntary, and questionnaire completion constituted consent. Human resource and administrative managers provided written informed consent for study participation. This study was approved by the ethics committee of the University of Occupational and Environmental Health, Japan.

SOC was assessed using a shortened version of Antonovsky's SOC questionnaire, the University of Tokyo Health Sociology version of the SOC scale (SOC-UTHS) $)^{7,8)}$. Responses to each question were scored on a Likert-type scale ranging from 1 to 7 , with higher scores indicating stronger SOC. In Japanese studies, the Cronbach's alpha of the SOC-UTHS was ranged from 0.82 to $0.84^{7}$, ${ }^{8)}$; furthermore, it was significantly associated with the SOC-13 ${ }^{8}$. Psychological distress was evaluated by the 28-item version of the General Health Questionnaire (GHQ) ${ }^{9)}$. The GHQ is a widely used tool for assessing psychological distress and screening for minor psychological disorders at work sites. Overtime work was defined as any working hours that exceeded normal daily hours $(8 \mathrm{~h}$ /day). Data about overtime work in the previous month were obtained from employees' computerized attendance records.

Age, gender, job position, job stress, and job support of the workers were considered potential confounding factors. Job stress and job support were calculated using the Brief Job Stress Questionnaire
(BJSQ), which is based on the demand-control-support model ${ }^{10)}$. The BJSQ was developed in Japan for occupational health check-ups, and it is widely used there. Twelve items of the BJSQ (a 57-item multidimensional questionnaire) were used to estimate job stress and job support. Job stress scores were calculated as job demand scores divided by job control scores (three items for each). Job support scores were the sum of the scores for the supervisor and coworker support subscales (three items for each).

\section{Statistical analysis}

All statistical calculations were performed using a statistical software package (JMP 9.0; SAS Institute Inc., Cary, NC, USA). To assess the buffering effect of SOC on the relationship between overtime work and mental health status, multiple regression analysis with mean-centered SOC scores was employed. Then, the interaction term-centered SOC $\times$ overtime work was included, followed by simple slope analysis to further explore the relationship. Then we used multiple regression analysis to adjust for potential confounding factors. All results are expressed as the mean \pm standard deviation $(S D)$. Values of $p<0.05$ were considered statistically significant.

\section{Results}

The survey was administered to a total of 1,558 employees (response rate: 93.5\%). The average age was $36.2 \pm 9.3$ years old. Females accounted for $19 \%$ of the subjects, and $21 \%$ of the subjects were in management job positions. The average scores of the GHQ, job stress, and job support were $7.3 \pm 6.0$, $16.1 \pm 3.7$, and $1.2 \pm 0.6$, respectively. The average SOC score was $15.0 \pm 3.4$, and the average number of hours of overtime work was $9.2 \pm 17.0 \mathrm{~h} / \mathrm{month}$. The internal consistency of the SOC-UTHS scale (Cronbach's $\alpha$ ) was 0.85 .

Multiple regression analysis revealed the main effects of overtime work $(\beta=0.08, p=0.0003)$ and SOC scores $(\beta=0.41, p<0.0001)$ on GHQ scores (Table $1)$. There was a tendency toward interaction between overtime work and SOC scores $(\beta=0.05, p=0.051$, Table 1).

Simple slopes for the association between overtime work and GHQ scores were tested for low (-1 SD below the mean) and high (+1 SD above the mean) levels of SOC scores (Table 1). As shown in Fig. 1, overtime work had a significant and positive correlation with GHQ scores when the SOC was low (simple slope $=0.04$, standard error $[S E]=0.01, p<0.0001$ ), but this relationship was not statistically significant when the SOC was high (simple slope=0.01, $S E=0.01$, $p=0.188)$.

Finally, we analyzed the relationship after adjust- 
Table 1. Multiple regression analysis

\begin{tabular}{|c|c|c|c|c|c|c|}
\hline Analysis model & Estimate & $\mathrm{SE}$ & $t$ value & $p$ value & & $\beta$ \\
\hline \multicolumn{7}{|l|}{ (1) Centering of SOC score without interaction } \\
\hline Intercept & 7.07 & 0.16 & 45.01 & $<0.0001$ & $* * *$ & 0 \\
\hline Overtime work & 0.03 & 0.01 & 3.59 & 0.0003 & $* * *$ & 0.08 \\
\hline SOC_C & -0.71 & 0.04 & 17.65 & $<0.0001$ & $* * *$ & 0.41 \\
\hline \multicolumn{7}{|l|}{ (2) Centering of SOC score with interaction } \\
\hline Intercept & 7.07 & 0.16 & 45.02 & $<0.0001$ & $* * *$ & 0 \\
\hline Overtime work & 0.03 & 0.01 & 3.63 & 0.0003 & $* * *$ & 0.08 \\
\hline SOC_C & -0.67 & 0.05 & 14.69 & $<0.0001$ & $* * *$ & 0.38 \\
\hline Overtime work $\times$ SOC_C & -0.004 & 0.002 & 1.96 & 0.051 & $\S$ & 0.05 \\
\hline \multicolumn{7}{|l|}{ (3) Simple slope analysis } \\
\hline \multicolumn{7}{|l|}{ Low SOC } \\
\hline Intercept & 9.37 & 0.22 & 42.08 & $<0.0001$ & $* * *$ & 0 \\
\hline Overtime work & 0.04 & 0.01 & 3.95 & $<0.0001$ & $* * *$ & 0.13 \\
\hline SOC_L & -0.67 & 0.05 & -14.69 & $<0.0001$ & $* * *$ & -0.38 \\
\hline Overtime work $\times$ SOC_L & -0.004 & 0.002 & -1.96 & 0.051 & $\S$ & -0.07 \\
\hline \multicolumn{7}{|l|}{ High SOC } \\
\hline Intercept & 4.77 & 0.22 & 21.62 & $<0.0001$ & $* * *$ & 0 \\
\hline Overtime work & 0.01 & 0.01 & 1.32 & 0.188 & & 0.04 \\
\hline SOC_H & -0.67 & 0.05 & -14.69 & $<0.0001$ & $* * *$ & -0.38 \\
\hline Overtime work $\times$ SOC_H & -0.004 & 0.002 & -1.96 & 0.051 & $\S$ & -0.07 \\
\hline \multicolumn{7}{|l|}{ (4) Adjusting for confounding factors } \\
\hline Intercept & 9.65 & 0.97 & 9.94 & $<0.0001$ & $* * *$ & 0 \\
\hline Overtime work & -0.003 & 0.007 & -0.42 & 0.673 & & -0.01 \\
\hline SOC_C & -0.42 & 0.04 & 9.94 & $<0.0001$ & $* * *$ & 0.24 \\
\hline Overtime work $\times$ SOC_C & -0.002 & 0.002 & 1.27 & 0.203 & & 0.03 \\
\hline Age & -0.08 & 0.02 & -4.68 & $<0.0001$ & $* * *$ & -0.12 \\
\hline Sex (male/female) & -0.23 & 0.16 & -1.42 & 0.156 & & -0.03 \\
\hline Job position (non-management/management) & -0.46 & 0.18 & -2.48 & 0.013 & $*$ & -0.06 \\
\hline Job support & -0.24 & 0.04 & -6.85 & $<0.0001$ & $* * *$ & -0.15 \\
\hline Job stress & 4.06 & 0.21 & 19.76 & $<0.0001$ & $* * *$ & 0.43 \\
\hline
\end{tabular}

SOC_C $=$ SOC score - average of SOC; SOC_L = SOC score - (average of SOC -1 SD); SOC_H = SOC score - (average of SOC + $1 \mathrm{SD}$ ); SOC, sense of coherence; SE, standard error; ${ }^{\circledR} p<0.1 ;{ }^{*} p<0.05 ; * * * p<0.0001$ Estimate, partial regression coefficient; $\beta$, standardized partial regression coefficient.

ing for age, gender, job position, job stress, and job support. Job stress showed the strongest association with GHQ scores $(\beta=0.43, p<0.0001)$, but there was no significant correlation between overtime work and GHQ scores $(\beta=-0.01, p=0.673$, Table 1$)$.

\section{Discussion}

SOC may buffer the association between objective overtime work (calculated with company payroll records) and mental health status. This is the first report using an objective index such as overtime work rather than subjective job quantity evaluated by questionnaire. However, after adjustment for confounding factors, there was no significant association between mental health status and overtime work. Thus, job stress might affect mental health status more than overtime work.

Previous research indicated that $\mathrm{SOC}$ acts as a buffer for health against the negative effects of workload when evaluated by self-reported questionnaires ${ }^{5,6,10)}$. Our data obtained using an index of objective workload indicated the safe finding. Notably, overtime work is multifaceted-on one hand, it results in higher job stress ${ }^{2}$, while on the other hand, it increases levels of participation in decisionmaking and opportunities to develop special abilities $^{11)}$. The present results suggest the importance of perceived stressors of workers, such as recognizing one's method of handling occupational stress, that is to say, SOC, in determining whether overtime work is 


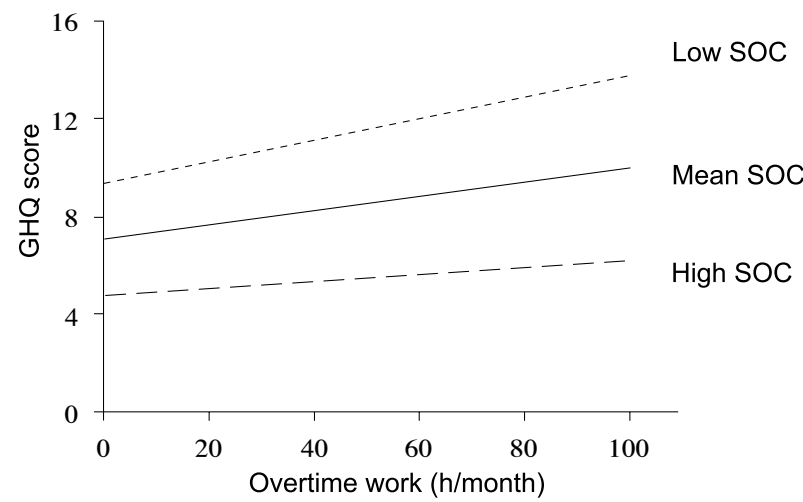

Fig. 1. Effect of overtime work on mental health status buffered by level of sense of coherence

The linear regression lines represent the predicted scores. All regression coefficients were set at 0 , except for overtime work and GHQ scores. Since we assume that the relationship between overtime work and GHQ scores is buffered by SOC scores, we illustrate this effect by predicting scores for 1 ) low SOC (defined as the mean SOC score minus $1 S D), 2$ ) the mean SOC, and 3) a high SOC (defined as the mean SOC score plus $1 S D)$. There is a trend toward interaction $(\beta=0.05, p=0.051)$. Simple slopes indicate that overtime work has a significant and positive correlation with GHQ scores at a low SOC and the mean SOC (low SOC, simple slope $=0.04, S E=0.01, p<0.0001$; mean SOC, simple slope $=0.03, S E=0.01, p=0.0003$ ). However, at above-average levels of SOC, this relationship is not statistically significant (high SOC, simple slope $=0.01, S E=0.01, p=0.188$ ).

a benefit or a hindrance.

Promoting SOC might be a good method of effectively warding against the adverse effects of job stress from overtime work. SOC can be enhanced by successful job experiences ${ }^{4}$, but specific interventions to promote SOC have rarely been suggested ${ }^{12)}$. Therefore, further research is needed. Occupational health staff should take care of workers who have a low SOC to prevent mental distress due to overtime work. Moreover, additional research must evaluate the best mental health-promoting environment for low SOC workers required to work overtime.

The strengths of this study include the objective assessment of overtime work. Furthermore, we had a high response rate, suggesting good reliability. Nevertheless, there are also some limitations. First, the short version of the SOC questionnaire was used in this study. Some studies have reported that the reliability of the short version was unacceptable (Cronbach's $\alpha=0.39-0.45)^{13,14)}$. However, our data showed an acceptable value $(\alpha=0.85)$. Furthermore, measuring SOC with only three items is recommended for large surveys with limited questionnaire space $^{8)}$.
Second, this study was conducted in one particular IT company in Japan, so the generalizability of our results to other populations is unknown. However, the present study's SOC scores were essentially equivalent to a representative sample survey's results (males, $15.0 \pm 3.5$; females, $14.9 \pm 3.3$ ) involving 4,800 Japanese people aged 20 to $40^{15}$. Third, crosssectional analyses do not clarify the causal relationships among the variables. Therefore, an important task in future studies will be to examine the role of SOC in well-being in longitudinal, wide-ranging business studies. In conclusion, strong SOC buffers the mental health impact of overtime work as measured by an objective index, and therefore should be considered when assessing the effects of workload on mental health.

Acknowledgments: This work was supported by a UOEH Grant for Advanced Research (2012) to MO.

Conflicts of interest: The authors have no conflicts of interest to declare.

\section{References}

1) Ministry of Health, Labour and Welfare. Creating Safe and Secure Workplaces (Chapter 3, Section 2) in Annual Health, Labour and Welfare Report 20072008. [Online]. 2008 [cited 2014 Oct 11]; Available from: URL: http://www.mhlw.go.jp/english/wp/ wp-hw2/part2/p2c3s2.pdf

2) Karhula K, Härmä M, Sallinen $M$, et al. Association of job strain with working hours, shift-dependent perceived workload, sleepiness and recovery. Ergonomics 2013; 56: 1640-51.

3) Hobson J, Beach JR. An investigation of the relationship between psychological health and workload among managers. Occup Med (Lond) 2000; 50: 518-22.

4) Antonovsky A. Unraveling the mystery of health: how people manage stress and stay well. San Francisco: Jossey-Bass; 1987.

5) Feldt $T$. The role of sense of coherence in wellbeing at work: analysis of main and moderator effects. Work Stress 1997; 11: 134-47.

6) Urakawa K, Yokoyama K. Sense of coherence (SOC) may reduce the effects of occupational stress on mental health status among Japanese factory workers. Ind Health 2009; 47: 503-8.

7) Haraoka T, Ojima T, Murata C, Hayasaka S. Factors influencing collaborative activities between non-professional disaster volunteers and victims of earthquake disasters. PLoS One 2012; 7: 1-8.

8) Togari T, Yamazaki Y, Nakayama K, Shimizu J. Development of a short version of the sense of coherence scale for population survey. J Epidemiol Community Health 2007; 61: 921-2.

9) Goldberg D. Manual of the General Health Questionnaire. N Berkshire (UK): NFER-Nelson; 
1978.

10) Urakawa K, Yokoyama K, Itoh H. Sense of coherence is associated with reduced psychological responses to job stressors among Japanese factory workers. BMC Res Notes 2012; 5: 247. (doi:10.118 6/1756-0500-5-247).

11) Grosch JW, Caruso CC, Rosa RR, Sauter SL. Long hours of work in the U.S.: associations with demographic and organizational characteristics, psychosocial working conditions, and health. Am J Ind Med 2006; 49: 943-52.

12) Mayer $\mathrm{CH}$, Boness $\mathrm{C}$. Interventions to promoting sense of coherence and transcultural competences in educational contexts. Int Rev Psychiatry 2011; 23: 516-24.

13) Olsson M, Gassne J, Hansson K. Do different scales measure the same construct? Three sense of coherence scales. J Epidemiol Community Health 2009; 63: $166-7$.

14) Schumann A, Hapke U, Meyer C, Rumpf HJ, John U. Measuring sense of coherence with only three items: a useful tool for population surveys. $\mathrm{Br} \mathrm{J}$ Health Psychol 2003; 8: 409-21.

15) Togari T. Development of the Useful SOC3 Scale (University of Tokyo Health Sociology Version of the SOC3 Scale: SOC3-UTHS) for the Large Population Survey. University of Tokyo Institute of Social Science Panel Survey. Discussion Paper Series 2008; No. 4. [Online]. 2008 [cited 2014 Oct 11]; Available from: URL: http://ssjda.iss.u-tokyo. ac.jp/panel/dp/PanelDP_004togari.pdf 